\title{
Treatment Patterns, Statin Intolerance, and Subsequent Cardiovascular Events Among Japanese Patients With High Cardiovascular Risk Initiating Statin Therapy
}

\author{
Saurabh P. Nagar; Pratik P. Rane, PhD; Kathleen M. Fox, PhD; \\ Juliana Meyers; Keith Davis; Anne Beaubrun, PhD; \\ Hyoe Inomata, MD, PhD; Yi Qian, PhD; Kouji Kajinami, MD
}

Background: This study examined treatment patterns, possible statin intolerance, and incidence of cardiovascular events (CVEs) in 2 cohorts of patients with high cardiovascular risk (i.e., patients with atherosclerotic cardiovascular disease [ASCVD] and patients with diabetes mellitus).

\begin{abstract}
Methods and Results: A retrospective cohort study examined adults initiating either a statin or ezetimibe from 1 January 2006 to 31 May 2014 in the Japan Medical Data Center database. The first observed statin or ezetimibe prescription defined the index date. Patients had $\geq 12$ months of pre- and post-index date plan enrollment. Two high-risk cohorts, the ASCVD cohort and diabetes cohort, were created based on diagnoses observed during the 12 months' pre-index date. Treatment patterns, possible statin intolerance, and incidence of CVEs were reported. In the ASCVD cohort $(n=5,302), 32.9 \%$ discontinued therapy, $7.7 \%$ switched to a non-index statin or non-statin lipid-lowering therapy, and $11.2 \%$ augmented index therapy in the 12 months' post-index date; only $0.3 \%$ were using high-intensity statins and 10\% had possible statin intolerance. Also, $8.1 \%$ had any new CVE during the follow-up period. Treatment patterns and incidence of CVEs among the diabetes cohort were similar to those of the ASCVD cohort.
\end{abstract}

Conclusions: High cardiovascular risk Japanese patients had frequent treatment modifications, although use of high-intensity statin doses was rare. These patterns may indicate that alternative therapies for lipid lowering are needed.

Key Words: Atherosclerotic cardiovascular disease; Cardiovascular event; Statins; Treatment patterns

$\mathbf{T}$ here has been a recent upward trend in the incidence of atherosclerotic cardiovascular disease (ASCVD), comprising peripheral artery disease (PAD), coronary artery disease, cerebrovascular atherosclerotic disease, and acute coronary syndrome (ACS), in the Japanese population. ${ }^{1-4}$ Japan Atherosclerotic Society guidelines for the prevention of ASCVD were developed in 2012 based on epidemiological and therapeutic evidence, with special care taken to address treatment pathways among patients considered at high-risk (e.g., patients with a history of ASCVD or patients with a history of diabetes)..$^{5}$ Specifically, in patients with a history of ASCVD, the guidelines recommend a combination of statins and eicosapentaenoic acid and/or ezetimibe. Guidelines also recommend that patients with diabetes and hyperlipidemia initiate treatment with statin therapy..$^{58}$

Currently, 6 statins are approved for use in Japan (i.e., atorvastatin, fluvastatin, pitavastatin, pravastatin, rosuvas-

\section{Editorial p 948}

tatin, and simvastatin). Previous studies have found that the Asian population is more sensitive to the effects of statins compared with the Western population, which has led to lower approved doses in Japan compared with the USA. $^{9-12}$ While generally well-tolerated, muscle, renal, and hepatic adverse events are occasionally observed in statin users. ${ }^{13-15}$ Additionally, concomitant medications may also increase the risk of adverse events such as muscle toxicity among statin users. ${ }^{16}$

Statin intolerance is defined as the inability to continue taking an effective dosage of statins due to adverse events. ${ }^{17}$ The 2013 American College of Cardiology and American Heart Association (ACC/AHA) joint guidelines recommend re-initiating statin treatment and/or switching to other lipid-lowering therapies (LLTs) for those who experience

Received August 6, 2017; revised manuscript received November 14, 2017; accepted November 27, 2017; released online December 23, 2017 Time for primary review: 26 days

RTI Health Solutions, Research Triangle Park, NC (S.P.N., J.M., K.D.); Amgen Inc., Thousand Oaks, CA (P.P.R., A.B., Y.Q.); Strategic Healthcare Solutions, LLC, Aiken, SC (K.M.F.), USA; Amgen Astellas BioPharma K. K., Tokyo (H.I.); and Department of Cardiology, Kanazawa Medical University, Ishikawa (K.K.), Japan

Mailing address: Kouji Kajinami, MD, Department of Cardiology, Kanazawa Medical University, 1-1 Daigaku, Uchinada, Ishikawa 920-0293, Japan. E-mail: kajinami@kanazawa-med.ac.jp

ISSN-1346-9843 All rights are reserved to the Japanese Circulation Society. For permissions, please e-mail: cj@j-circ.or.jp 
statin intolerance. ${ }^{18}$ However, statin intolerance and/or ineffectiveness could limit the potential ASCVD risk reduction of statin treatment. ${ }^{\mathbf{1 9 , 2 0}}$

While previous studies conducted in Japan have suggested that early treatment with atorvastatin may be associated with a lower risk of major adverse cardiac and cerebrovascular events in patients with a history of ASCVD, ${ }^{21}$ limited published data exist from Japanese populations on the incidence of cardiovascular events (CVEs) among high-risk patients with a history of ASCVD or diabetes. To fill the evidence gap, this study examined treatment patterns and possible statin intolerance/ineffectiveness in 2 cohorts of high-risk patients initiating statins and/or ezetimibe treatment: (1) patients with ASCVD; and (2) patients with type 2 diabetes. This study also investigated the incidence of CVEs for each cohort overall and for the subgroups of patients in each cohort who had statin dose up-titration.

\section{Methods}

This was a retrospective cohort analysis using data from the Japan Medical Data Center (JMDC) between January 1, 2005, and May 31, 2015. Japanese patients with ASCVD or type 2 diabetes initiating statin or ezetimibe therapy were identified, and treatment patterns, possible statin intolerance/ineffectiveness, and the incidence of CVEs were evaluated.

\section{Data Source}

The JMDC database comprises retrospective claims data sourced from the Japanese union-managed health insurance system (Health Insurance Association). The database includes information predominantly from persons of working age (i.e., less than 65 years old) employed by middle- to large-size companies as well as their dependents. The database currently includes more than 1 million unique persons from 2003 onward, and represents approximately $1 \%$ of the total population of Japan. Data elements captured in the JMDC database include patient-level demographic and plan enrollment information, inpatient and outpatient medical claims, and pharmacy claims. The study data spanned a longitudinal period of 10 years, from January 1, 2005, through May 31, 2015. As data were retrospective, deidentified, and anonymous, RTI International's institutional review board committee determined that this study did not constitute research with human subjects and was therefore exempt from institutional review board consideration.

\section{Patient Selection Criteria and Study Cohorts}

Patients were selected for inclusion in the study if they had at least 1 pharmacy claim for a statin or ezetimibe between January 1, 2006, and May 31, 2014. The date of the first statin or ezetimibe prescription defined the index date, and patients were required to be at least 18 years of age at the index date. Additionally, patients were required to have at least 12 months of continuous health plan enrollment pre- and post-index date. Patients were excluded from the analysis if they received multiple statins on the index date or had a pharmacy claim for either a statin or ezetimibe in the 12 months' pre-index date. Patients initiating both statin and ezetimibe on the index date were included in the study. Finally, patients were required to qualify for 1 of 2 non-mutually exclusive, high-risk cohorts based on diagnoses observed during the 12 months' pre-index date: (1) the ASCVD cohort; or (2) the diabetes cohort.

Patients were selected for inclusion in the ASCVD cohort if they had at least 1 diagnosis claim of myocardial infarction (MI), unstable angina (UA), coronary artery bypass graft (CABG), percutaneous coronary intervention (PCI), ischemic stroke (IS), transient ischemic attack (TIA), or PAD during the 12 months' pre-index period. Patients were selected for inclusion in the diabetes cohort if they did not have diagnosis for MI, UA, CABG, PCI, IS, TIA, or PAD during the 12 months' pre-index date, but did have a diagnosis of type 2 diabetes during this time period.

\section{Study Measures}

The demographic characteristics, including age and sex, that were available in patients' claims data were documented. The presence of cardiovascular disease (CVD)-related comorbidities (i.e., MI, UA, CABG, PCI, IS, PAD, diabetes mellitus, abdominal aortic aneurysm, and angina pectoris) and the Charlson Comorbidity Index (CCI) score - as defined by International Statistical Classification of Diseases and Related Health Problems, 10th Revision (ICD-10) diagnosis codes - observed during the 12 months' pre-index period were assessed.

Treatment characteristics (i.e., types of LLTs received on the index date, statin dosage on the index date, discontinuation, switching, augmentation, and dose titration) were assessed. Discontinuation, switching, augmentation, and dose titration were evaluated from the index date until the earlier of either the date of health plan disenrollment ( $\geq 12$ months from index date) or the end of the study period (May 31, 2015). Statin dose was calculated by multiplying the quantity of medication supplied by the dose and dividing by the days of supply. Additional LLTs that patients may have received upon switching or augmenting treatment included ezetimibe (among patients with statin only as their index medication), niacin, bile-acid resin, other fibric acid derivatives, cholesterol absorption inhibitors, or statins (among patients with ezetimibe only as their index medication).

Discontinuation was defined as the date of the first observed index medication refill gap of at least 90 days. The proportion of patients with discontinuation and the time to discontinuation (from index date) were documented. Discontinuation was classified as "permanent" (i.e., no reuptake of the index medication observed for the duration of available follow up) or "temporary" (i.e., re-initiated the index medication at any point after the 90-day minimum refill gap).

Switching was defined as initiation of a new prescription for another LLT (i.e., a non-index statin or non-statin LLT) without continued use of the index medication. The proportion of patients with a switch, the distribution of alternative agent(s) switched to, and the time from index date to switch were assessed. Switching to a non-index statin was evaluated separately from switching to a non-statin LLT.

Augmentation was defined as the start of an additional LLT with continued use of the index treatment regimen. To classify as augmentation, patients were required to have an overlapping days' supply of $\geq 60$ days for both the index treatment regimen and the additional LLT. The proportion of patients who augmented and class of medication augmented with were evaluated along with the time from the index date to augmentation of therapy.

Statin dose titration, among patients initiating statin 


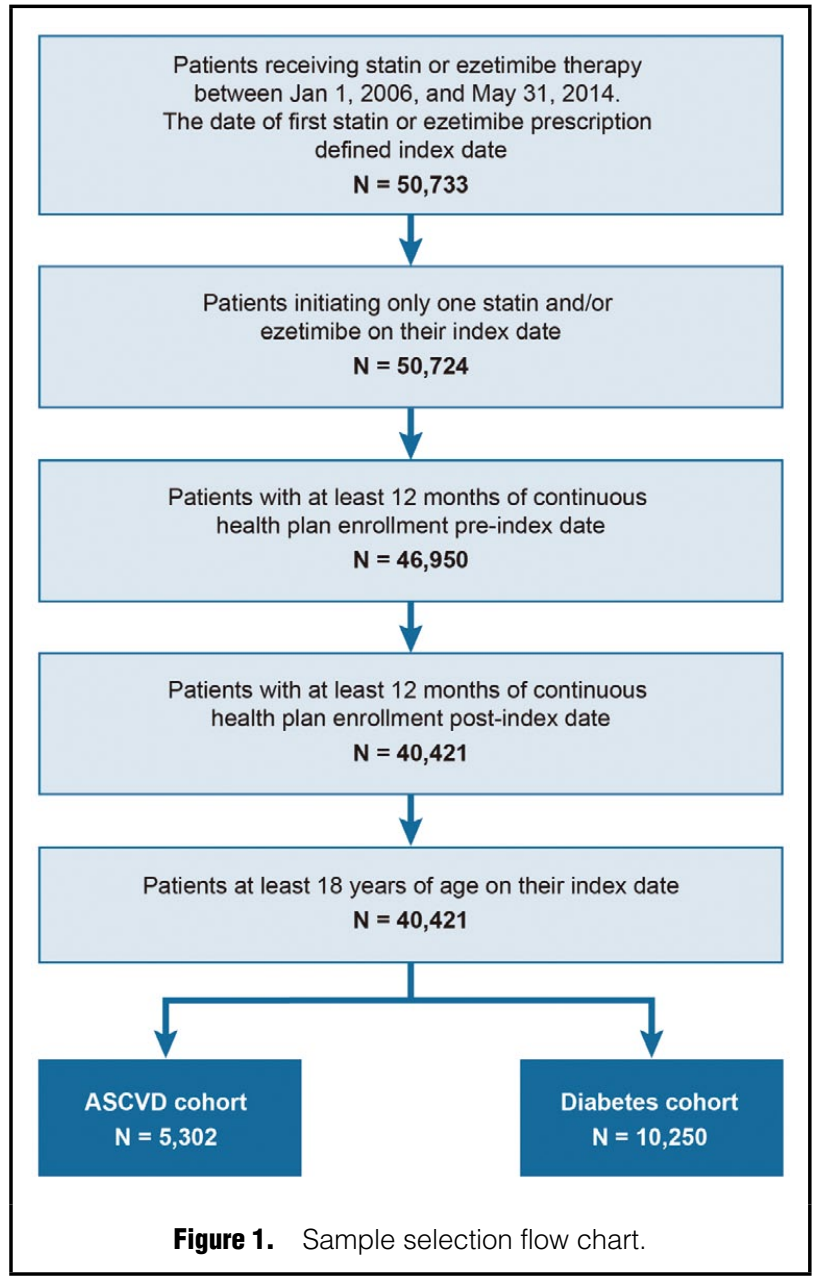

treatment (alone or with ezetimibe) on the index date, was defined as a change of at least $25 \%$ of the index statin dose, with both up-titration (i.e., an increase of $\geq 25 \%$ of the index dose) and down-titration (i.e., a decrease of $\geq 25 \%$ of the index dose) reported. The percentage of patients who titrated to the maximum recommended statin dose was reported along with the time from the index date to titration to the maximum recommended statin dose. Dose intensities (i.e., low intensity, moderate intensity, greater-thanmoderate intensity) based on modified definitions of the 2013 ACC/AHA guidelines were reported. ${ }^{22}$

Adherence to statin or ezetimibe therapy was reported as the medication possession ratio (MPR), defined as the proportion of patients' time on the drug (days between the index prescription and the end of a 12-month post-index date period) with medication supply on hand. Compliance to statin or ezetimibe therapy was defined as the number and percentage of patients with a MPR $\geq 80 \%$. Persistence was defined as the percentage of patients who remained on therapy for the duration of available follow up with no gap greater than 60 days.

Intolerance and/or ineffectiveness issues were reported and were proxied based on a previously developed algorithm. ${ }^{23}$ Specifically, using the combination of the first and second observed treatment modification, the number and percentage of patients with possible statin tolerability issues, possible statin tolerability and/or ineffectiveness, possible statin ineffectiveness, and possible non-statin tolerability and/or ineffectiveness were reported.

Indicators of possible statin intolerance included statin dose reduction, temporary discontinuation followed by re-initiation of the same statin, and subtracting the statin from a combination therapy regimen. Indicators of possible statin intolerance and/or ineffectiveness included switching to a different statin or discontinuation of statin therapy. Indicators of possible statin ineffectiveness included dose escalation $(\geq 25 \%)$ and augmentation with a non-statin LLT. Indicators of possible non-statin intolerance and/or ineffectiveness included switching to a statin from ezetimbe or discontinuation of ezetimibe.

Incident CVEs (i.e., defined as an inpatient claim of MI, UA, IS, TIA, CABG, heart failure [HF], and PAD) were reported by study cohort as well as among the subgroups of patients in each cohort with statin dose up-titration by $\geq 25 \%$. For each cohort, CVEs were evaluated from the index date to the earlier of either disenrollment from the health plan or the end of the study (March 2015). For the subgroups of patients in each cohort with statin dose uptitration, CVEs were evaluated from the date of the first titration to the earlier of either disenrollment from the health plan or the end of the study. This subgroup analysis was of interest to determine if patients on higher doses of statins (i.e., patients who up-titrated their dose) had a lower rate of new CVEs in comparison to the overall cohort. If multiple CVEs were observed, only one CVE was reported based on the clinical importance (e.g., acute/urgency) according to the following hierarchy: MI, UA, IS, TIA, $\mathrm{CABG}, \mathrm{HF}$, and PAD.

\section{Data Analysis}

Study outcomes, including treatment characteristics and dose intensity, statin intolerance/ineffectiveness, and CVEs were reported as mean values, medians, ranges, and standard deviations (SDs) for continuous variables, and frequency distributions for categorical variables. Incidence rates of CVEs per 1,000 person-years of follow up were reported. All analyses were performed using SAS statistical software, version 9.4.

\section{Results}

Between January 1, 2006 and May 31, 2014, there were 50,724 patients aged at least 18 years old initiating either statin or ezetimibe therapy who did not initiate multiple statins on the same day (Figure 1). From this population, 40,421 patients had at least 12 months' pre- and post-index date continuous health plan enrollment, with 5,302 (13.1\%) patients in the ASCVD cohort and 10,250 (25.4\%) patients in the diabetes cohort.

Among patients in the ASCVD and diabetes cohorts, the mean (SD) age was 55.7 (9.5) and 52.8 (9.5) years, respectively, and in both cohorts, approximately two-thirds were male (Table 1). In both cohorts, approximately twothirds of patients had a hyperlipidemia diagnosis during the 12 months' pre-index date. Diabetes mellitus and hypertension were the most common CVD-related comorbidities among patients in the ASCVD cohort; hypertension and PAD were the most common CVD-related comorbidities among patients in the diabetes cohort.

Among patients in both the ASCVD and diabetes cohorts, rosuvastatin and atorvastatin were the most common statins received on the index date (Table 2). 


\begin{tabular}{|c|c|c|c|c|}
\hline \multirow{2}{*}{$\begin{array}{l}\text { Characteristics } \\
\text { Age at index date, years (mean [SD]) }\end{array}$} & \multicolumn{2}{|c|}{$\begin{array}{l}\text { ASCVD cohort } \\
(n=5,302)\end{array}$} & \multicolumn{2}{|c|}{$\begin{array}{c}\text { Diabetes cohort } \\
\quad(n=10,250)\end{array}$} \\
\hline & 55.7 & 9.5 & 52.8 & 9.5 \\
\hline Male ( $n$, column \%) & 3,385 & 63.8 & 6,626 & 64.6 \\
\hline \multicolumn{5}{|l|}{ Comorbidities (n, column \%) } \\
\hline Hyperlipidemia & 3,437 & 64.8 & 7,008 & 68.4 \\
\hline Myocardial infarction & 1,150 & 21.7 & - & 0.0 \\
\hline Unstable angina & 281 & 5.3 & - & 0.0 \\
\hline Coronary artery bypass graft & 264 & 5.0 & - & 0.0 \\
\hline Percutaneous coronary intervention & 264 & 5.0 & - & 0.0 \\
\hline Ischemic stroke or transient ischemic attack & 1,944 & 36.7 & - & 0.0 \\
\hline Peripheral artery disease & 2,759 & 52.0 & - & 11.1 \\
\hline Diabetes mellitus & 3,160 & 59.6 & 10,250 & 100.0 \\
\hline Hypertension & 3,152 & 59.5 & 4,874 & 47.6 \\
\hline Abdominal aortic aneurysm & 70 & 1.3 & - & 0.0 \\
\hline Angina pectoris & 1,538 & 29.0 & 1,040 & 10.2 \\
\hline CCl score (mean [SD]) & 2.7 & 1.9 & 2.1 & 1.4 \\
\hline
\end{tabular}

ASCVD, atherosclerotic cardiovascular disease; CCI, Charlson Comorbidity Index; SD, standard deviation.

Table 2. (A) Statin Dose Characteristics and Titration in the Follow-up Period, (B) Treatment Patterns in the Follow-up Period: Duration of Discontinuation, Switching, and Augmentation

\section{Characteristic}

A

Medications received on the index date ( $n$, column \%)

Atorvastatin

Fluvastatin

Pitavastatin

Pravastatin

Rosuvastatin

Simvastatin

Ezetimibe

Both statin and ezetimibe

Index statin dose intensity (n, column \%)

Low intensity ${ }^{A}$

Moderate intensity ${ }^{B}$

Greater than moderate intensityc

Patients with a statin dose change in the 1-year post-index period

Upward titration ( $n$, column \%)

Upward titration to the maximum dose ( $\mathrm{n}$, column \%)

Time (days) from statin initiation to titration to maximum dose (mean [SD])

B

Time to discontinuation, mean (SD)

Time to switching, mean (SD)

Time to augmentation, mean (SD)

$\begin{array}{cc}\underset{(n=5,302)}{\text { ASCVD cohort }} & \text { Diabetes cohort } \\ (n=10,250)\end{array}$

$(n=5,302)$

$(n=10,250)$

ASCVD, atherosclerotic cardiovascular disease; SD, standard deviation. Note: Patients with outlier doses were excluded from this analysis $(n=329$ in ASCVD cohort; $n=804$ in diabetes cohort). ALow-intensity doses include atorvastatin $2.5 \mathrm{mg}$, fluvastatin 10-30 mg, pitavastatin $0.5-1 \mathrm{mg}$, pravastatin $2.5-20 \mathrm{mg}$, rosuvastatin $1.25 \mathrm{mg}$, and simvastatin $2.5-10 \mathrm{mg}$. BModerate-intensity doses include atorvastatin $5-20 \mathrm{mg}$, pitavastatin $2-4 \mathrm{mg}$, and rosuvastatin $2.5-5 \mathrm{mg}$. ${ }^{\mathrm{C}}$ Greater than moderate-intensity doses include atorvastatin $>20 \mathrm{mg}$, fluvastatin $40-60 \mathrm{mg}$, rosuvastatin $>5 \mathrm{mg}$, and simvastatin $15-20 \mathrm{mg}$.

Ezetimibe alone was received by $5.5 \%$ and $7.1 \%$ of patients in the ASCVD and diabetes cohorts, respectively, and an ezetimibe and statin combination was received by less than $1 \%$ of patients in the ASCVD and diabetes cohorts. Among patients in the ASCVD and diabetes cohorts, very few statin users had greater-than-moderate intensity doses at the index date (Table 2). In total, $7.7 \%$ of patients in the ASCVD cohort and $6.2 \%$ of patients in the diabetes cohort experienced dose up-titration.

In the 12 months' post-index date, one-third of patients 


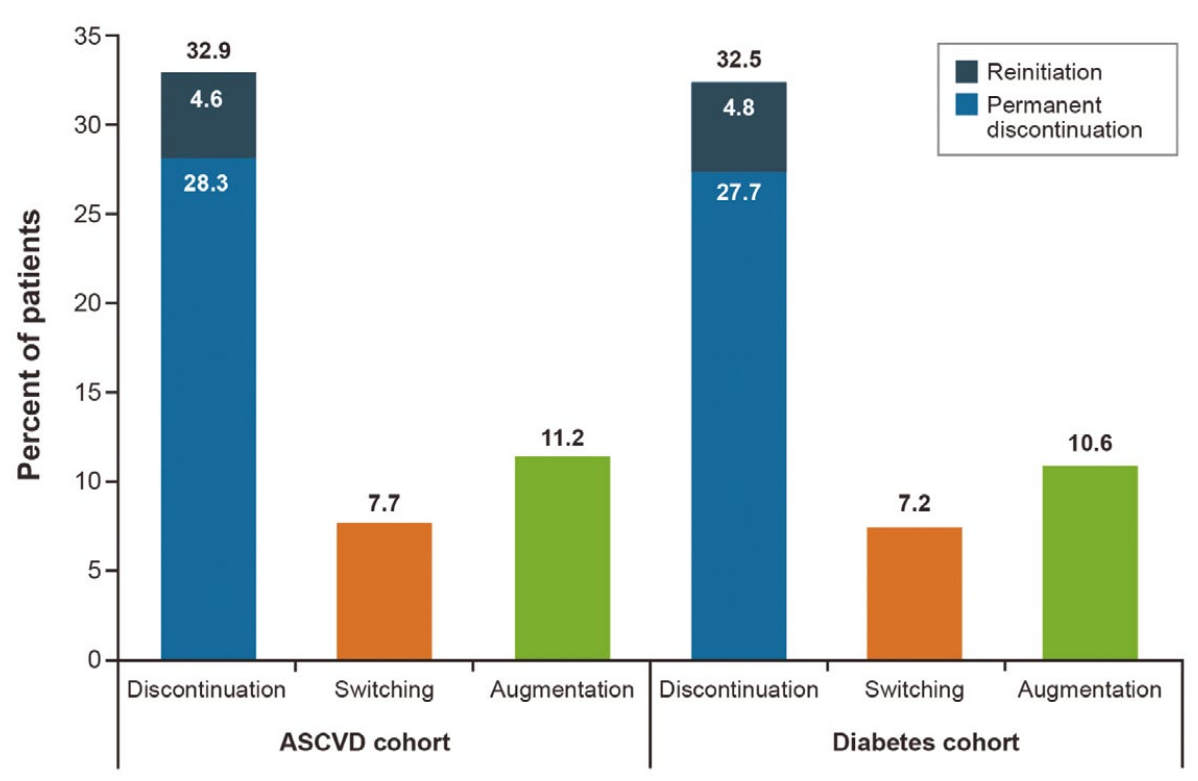

High risk patient cohorts

Figure 2. Treatment patterns in the 12-months follow-up period: percentage of patients discontinuing, switching, or augmenting treatment. ASCVD, athersclerotic cardiovascular disease.

in the ASCVD cohort discontinued either statin or ezetimibe treatment (Figure 2). Among those patients in the ASCVD cohort who discontinued index treatment, the majority discontinued statin or ezetimibe usage permanently with no re-initiation. Among patients in the ASCVD cohort with discontinuation, mean (SD) time from the index date to the date of discontinuation was 93.3 (74.1) days (Table 2). A total of $5.6 \%$ of patients in the ASCVD cohort switched to a non-index statin and $2.1 \%$ switched to a non-statin LLT (data not presented). Among patients in the ASCVD cohort who switched therapy, the mean (SD) time to switch was $107.6(102.2)$ days. A total of $11.2 \%$ of patients in the ASCVD cohort augmented index statin or ezetimibe with a non-statin LLT. Among patients in the ASCVD cohort who augmented therapy, the mean (SD) time to augmentation was 156.1 (80.0) days.

In the 12 months' post-index date, one-third of patients in the diabetes cohort discontinued either statin or ezetimibe treatment (Figure 2). Among those patients in the diabetes cohort who discontinued index treatment, the majority discontinued statin or ezetimibe permanently with no reinitiation. Among patients in the diabetes cohort with discontinuation, the mean (SD) time from the index date to the date of discontinuation was 100.4 (73.9) days (Table 2). A total of $4.9 \%$ of patients in the diabetes cohort switched to a non-index statin and $2.3 \%$ switched to a non-statin LLT. Among patients in the diabetes cohort who switched therapy, the mean (SD) time to switch was $120.0(99.4)$ days. A total of $10.6 \%$ of patients in the diabetes cohort augmented with a non-statin LLT. Among patients in the diabetes cohort who augmented therapy, the mean (SD) time to augmentation was 160.2 (78.5) days.

Among patients in the ASCVD cohort, almost two-thirds of patients were persistent to their index therapy (Table 3). The mean (SD) MPR for patients with statins as their index treatment was $0.8(0.3)$, with nearly three-quarters of patients achieving an MPR $\geq 0.80$. The mean (SD) MPR for patients with ezetimibe as their index treatment was 0.8 (0.3), with $71.1 \%$ of patients achieving an MPR $\geq 0.80$.

Similarly, among patients in the diabetes cohort, almost two-thirds of patients were persistent to their index therapy (Table 3). The mean (SD) MPR for patients in the diabetes cohort with statins as their index treatment was $0.8(0.3)$, with nearly three-quarters of patients achieving an MPR $\geq 0.80$. The mean (SD) MPR for patients in the diabetes cohort with ezetimibe as their index treatment was $0.8(0.3)$, with $69.2 \%$ of patients achieving an MPR $\geq 0.80$.

Possible statin intolerance was observed in $10.0 \%$ of patients in the ASCVD cohort, and $5.4 \%$ of patients in the ASCVD cohort were identified as having possible statin ineffectiveness or intolerance (Figure 3). A total of $7.8 \%$ of patients in the ASCVD cohort could be identified as having possible statin ineffectiveness, and possible non-statin intolerance or ineffectiveness was observed in $7.1 \%$ of patients in the ASCVD cohort.

Possible statin intolerance was observed in $8.2 \%$ of patients in the diabetes cohort, and $4.6 \%$ of patients in the diabetes cohort were identified as having possible statin ineffectiveness or intolerance. A total of $6.5 \%$ of patients in the diabetes cohort could be identified as having possible statin ineffectiveness, and possible non-statin intolerance or ineffectiveness was observed in $5.1 \%$ of patients in the diabetes cohort.

Among patients in the ASCVD cohort, 8.1\% experienced a new CVE between their index date and the end of the follow-up period (mean follow up of 2.8 years); MI, IS, and $\mathrm{HF}$ were the most common CVEs during this time period (Table 4). Incidence of the first CVE per 1,000 person-years of follow up was 29.5. A total of $15.4 \%$ of patients in the ASCVD cohort had a statin dose up-titration 


\begin{tabular}{|c|c|c|c|c|}
\hline \multirow{2}{*}{$\begin{array}{l}\text { Characteristic } \\
\text { Persistent (n, column \%) } \\
\text { Patients persistent on statin treatment }\end{array}$} & \multicolumn{2}{|c|}{$\begin{array}{l}\text { ASCVD cohort } \\
(n=5,302)\end{array}$} & \multicolumn{2}{|c|}{$\begin{array}{l}\text { Diabetes cohort } \\
\quad(n=10,250)\end{array}$} \\
\hline & 3,481 & 61.0 & 6,792 & 63.4 \\
\hline Patients persistent on ezetimibe treatment & 197 & 60.8 & 481 & 60.0 \\
\hline $\begin{array}{l}\text { Statin MPR-overall (mean [SD])A } \\
\text { Categories }(\mathrm{n} \text {, column \%) }\end{array}$ & 0.82 & 0.29 & 0.81 & 0.28 \\
\hline$\geq 0.80$ & 3,742 & 74.7 & 6,855 & 72.0 \\
\hline $0.79-0.70$ & 308 & 6.1 & 710 & 7.5 \\
\hline $0.69-0.60$ & 189 & 3.8 & 401 & 4.2 \\
\hline $0.59-0.50$ & 163 & 3.3 & 358 & 3.8 \\
\hline $0.49-0.40$ & 98 & 2.0 & 238 & 2.5 \\
\hline $0.39-0.30$ & 67 & 1.3 & 134 & 1.4 \\
\hline $0.29-0.20$ & 47 & 0.9 & 101 & 1.1 \\
\hline$<0.20$ & 399 & 8.0 & 726 & 7.6 \\
\hline $\begin{array}{l}\text { Ezetimibe MPR-overall (mean [SD]) } \\
\text { Categories ( } \mathrm{n} \text {, column \%) }\end{array}$ & 0.78 & 0.32 & 0.79 & 0.30 \\
\hline$\geq 0.80$ & 234 & 71.1 & 554 & 69.2 \\
\hline $0.79-0.70$ & 19 & 5.8 & 69 & 8.6 \\
\hline $0.69-0.60$ & 13 & 4.0 & 37 & 4.6 \\
\hline $0.59-0.50$ & 14 & 4.3 & 27 & 3.4 \\
\hline $0.49-0.40$ & 2 & 0.6 & 18 & 2.3 \\
\hline $0.39-0.30$ & 4 & 1.2 & 10 & 1.3 \\
\hline $0.29-0.20$ & 3 & 0.9 & 7 & 0.9 \\
\hline$<0.20$ & 40 & 12.2 & 79 & 9.9 \\
\hline
\end{tabular}

ASCVD, atherosclerotic cardiovascular disease; MPR, medication possession ratio; SD, standard deviation. APatients on statins or statin+ezetimibe combination only ( $n=5,013$ in ASCVD cohort; $n=9,521$ in diabetes cohort). BPatients on ezetimibe or statin+ezetimibe combination only ( $n=329$ in ASCVD cohort; $n=801$ in diabetes cohort).

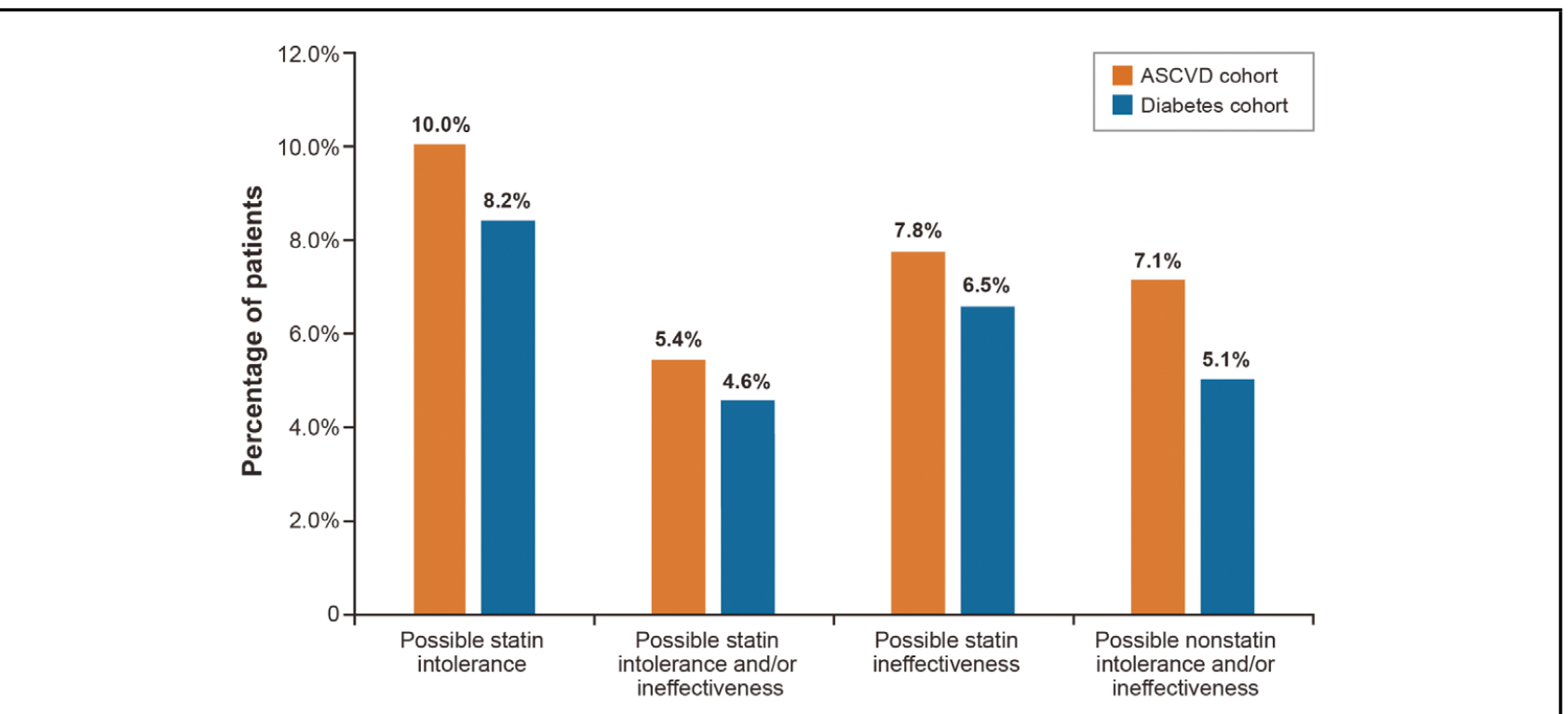

Figure 3. Statin intolerance and/or ineffectiveness issues in the follow-up period. ASCVD, atherosclerotic cardiovascular disease.

in the 12 months' post-index date. Among patients in the ASCVD cohort with statin dose up-titration, 8.6\% experienced a new CVE between the date of their first titration and the end of the follow-up period (mean follow up of 2.3 years); MI, IS, and HF were the most common CVEs during this time period. Incidence of the first CVE among patients with ASCVD with statin dose up-titration per 1,000 person-years of follow up was 31.9.

Among patients in the diabetes cohort, $2.6 \%$ experienced a new CVE between their index date and the end of the 


\begin{tabular}{|c|c|c|c|c|c|c|c|c|}
\hline \multirow{2}{*}{ Characteristic } & \multicolumn{4}{|c|}{ All patients } & \multicolumn{4}{|c|}{$\begin{array}{l}\text { All patients with statin dose up-yitration } \\
\qquad(\geq 25 \% \text { increase })\end{array}$} \\
\hline & \multicolumn{2}{|c|}{$\begin{array}{l}\text { ASCVD cohort } \\
(n=5,302)\end{array}$} & \multicolumn{2}{|c|}{$\begin{array}{l}\text { Diabetes cohort } \\
\quad(n=10,250)\end{array}$} & \multicolumn{2}{|c|}{$\begin{array}{l}\text { ASCVD cohort } \\
(n=385)\end{array}$} & \multicolumn{2}{|c|}{$\begin{array}{c}\text { Diabetes cohort } \\
\quad(n=588)\end{array}$} \\
\hline \multicolumn{9}{|l|}{ Type of event (n, column \%) } \\
\hline Any event & 431 & 8.1 & 263 & 2.6 & 33 & 8.6 & 29 & 4.9 \\
\hline Myocardial infarction & 115 & 2.2 & 57 & 0.6 & 11 & 2.9 & 9 & 1.5 \\
\hline Stroke & 125 & 2.4 & 63 & 0.6 & 9 & 2.3 & 7 & 1.2 \\
\hline Coronary revascularization procedure & 25 & 0.5 & 8 & 0.1 & 1 & 0.3 & - & 0.0 \\
\hline Heart failure on an inpatient claim & 80 & 1.5 & 85 & 0.8 & 6 & 1.6 & 8 & 1.4 \\
\hline Transient ischemic attack & 4 & 0.1 & - & 0.0 & - & 0.0 & - & 0.0 \\
\hline Unstable angina & 40 & 0.8 & 20 & 0.2 & 4 & 1.0 & 3 & 0.5 \\
\hline Peripheral artery disease & 42 & 0.8 & 30 & 0.3 & 2 & 0.5 & 2 & 0.3 \\
\hline \multicolumn{9}{|l|}{$\begin{array}{l}\text { Incidence of first cardiovascular event } \\
\text { per } 1,000 \text { person-years of follow up }\end{array}$} \\
\hline Any event & 29.5 & & 9.3 & & 31.9 & & 17.8 & \\
\hline Myocardial infarction & 7.9 & & 2.0 & & 10.6 & & 5.5 & \\
\hline Stroke & 8.6 & & 2.2 & & 8.7 & & 4.3 & \\
\hline Coronary revascularization procedure & 1.7 & & 0.3 & & 1.0 & & 0.0 & \\
\hline Heart failure on an inpatient claim & 5.5 & & 3.0 & & 5.8 & & 4.9 & \\
\hline Transient ischemic attack & 0.3 & & 0.0 & & 0.0 & & 0.0 & \\
\hline Unstable angina & 2.7 & & 0.7 & & 3.9 & & 1.8 & \\
\hline Peripheral artery disease & 2.9 & & 1.1 & & 1.9 & & 1.2 & \\
\hline
\end{tabular}

ASCVD, atherosclerotic cardiovascular disease.

follow-up period (mean follow up of 2.8 years); MI, IS, and HF were the most common CVEs during this time period (Table 4). Incidence of the first CVE in the diabetes cohort per 1,000 person-years of follow up was 9.3. A total of $26.8 \%$ of diabetes patients had a statin dose up-titration in the post-index date period. Among patients in the diabetes cohort with dose titration, $4.9 \%$ experienced a new CVE between the date of their statin dose up-titration and the end of the follow-up period (mean follow up of 2.4 years); MI, IS, and HF were the most common CVEs during this time period. Incidence of the first CVE in the diabetes cohort with statin dose up-titration per 1,000 person-years of follow up was 17.8 .

\section{Discussion}

In this retrospective observational study, treatment patterns, dose intensities, and potential statin intolerance and/or ineffectiveness were assessed among high CVD risk patients in Japan initiating treatment with either statins and/or ezetimibe. Results from the current study showed that a considerable proportion (33\%) of high-risk patients discontinued statin/ezetimibe therapy within fewer than 100 days, and use of high-intensity statin doses was rare $(<0.4 \%)$. Possible explanations for the high rate of discontinuation observed in the current analysis may be that patients were either not achieving a low-density lipoprotein cholesterol (LDL-C) goal with their low-to-moderate intensity statin therapy and/or patients were experiencing tolerability problems. The current study also reported that almost $10 \%$ of patients in the high-risk cohorts of patients with ASCVD and patients with diabetes had statin intolerance during the follow-up period. A retrospective study con- ducted in Asian countries reported lower LDL-C goal attainment among Asians in high-risk groups receiving statins compared with low-risk groups. ${ }^{24}$ Furthermore, Teramoto et al (2005) reported that the majority $(>70 \%)$ of high-risk patients on statin therapy in Japan did not achieve the lipid management goals recommended by the Japan Atherosclerotic Society in 2002.25,26 Results from our analysis highlight the issue of inadequate statin dosing, as almost all high-risk patients received low-to-moderate doses and approximately one-third of patients discontinued treatment. Thus, receipt of inadequate statin dose and the high need for subsequent treatment intensification observed in our analysis also highlight both the need for better physician understanding of intensive LDL-C treatment and alternative therapeutic options for the high-risk patients in Japan.

Adverse events (e.g., musculoskeletal, insomnia, peripheral neuropathy, and creatinine phosphokinase) associated with LLT could also influence treatment decisions, thereby increasing the likelihood of statin therapy discontinuation. In the current study, a considerable proportion of patients had a possible statin intolerance; therefore, the possibility of statin therapy discontinuation due to dose-related adverse events was high. Results from the current study suggested high adherence rates for statin therapy among high-risk groups. This was consistent with current literature showing a mean MPR of 0.9 among Japanese patients with highrisk vascular disease (HRVD) on statin therapy. ${ }^{27}$ Direct comparisons, however, between the two studies should be made with caution because ASCVD and HRVD definitions were not similar between the two studies, and study designs were not completely aligned, specifically with duration of insurance coverage post ASCVD/HRVD date. 
The current study also explored the incidence of CVEs among patients with high cardiovascular risk on statin therapy in Japan. Results suggest that many patients with ASCVD (8.1\%) still had subsequent CVEs despite receiving statins or ezetimibe. Among those patients with ASCVD who had an upward statin dose titration in the post-index date period, the proportion with subsequent CVEs was $8.6 \%$ from the first observed titration until the end of follow up. The incidence rates were similar for overall high-risk ASCVD and the dose up-titration subgroup. However, the incidence rates were slightly lower for the overall high-risk diabetes cohort compared with the dose up-titration subgroup of the high-risk diabetes cohort. The similar incidence rates for CVEs observed between patients with ASCVD overall and patients with ASCVD with dose up-titration may be due to factors such as lower proportion of high-risk patients with dose up-titration and lesser follow-up time for patients with dose up-titration, which could have underestimated the true rates of CVEs. Further research is needed in larger populations of patients with dose uptitration to confirm these findings.

The findings regarding the incidence of CVEs are consistent with existing literature that showed that $6.9 \%$ of high-risk patients enrolled in the Global Reduction of Atherothrombosis for Continued Health registry conducted in 29 countries including Japan experienced at least one event of MI or IS in the follow-up period. ${ }^{28}$ Another study using data from the Prevention of Atherothrombotic Incidents Following Ischemic Coronary Attack registry conducted among Japanese ACS patients reported that $6.4 \%$ of the patients with ACS had a new major adverse cardiac or cerebrovascular event in the follow-up period. ${ }^{29}$ These results further add to the earlier point that increasing the statin dose may not be sufficient to lower the risk of CVEs and other therapeutic options, and that other risk factor controls (e.g., hypertension, obesity) are needed to reduce the cardiovascular burden among high-risk patients in Japan.

Findings from the current study should be considered within the context of several limitations inherent in the JMDC data source. First, the study sample was selected from persons with healthcare coverage by the Japanese union-managed health insurance system, which may not be representative of all persons in Japan who receive healthcare benefits under other insurance types. Also, because most enrollees are working adults or their family members, the proportion of elderly patients aged 65 years and older is low, and the elderly patients in the database work force tend to be healthier than the general elderly population. The JMDC database contains only the month and year of service at the claim level for inpatient and outpatient encounters. Therefore, it was necessary to assume a withinmonth service date (i.e., last day of the month) for analytic purposes, which may have influenced the accuracy of event time and other duration measures. No information on lab test results was available; therefore, assessments of LDL-C values were not possible. Furthermore, the diagnoses recorded in the claims data were subject to administrative coding errors and could not be validated with a post-hoc medical record review. To our knowledge, there is currently no existing, validated claims-based algorithm to identify statin intolerance issues. We used a claims-based algorithm by Quek et al (2015) to identify statin intolerance and/or ineffectiveness issues based on pharmacy claims. ${ }^{23}$ No information was available in the database regarding reasons for treatment modification or the cause of statin intolerance. Further research (e.g., clinical chart review) is warranted to validate the statin intolerance and/or ineffectiveness algorithm. Finally, information was available only on prescription claims filled, and it was not possible to know whether the patient took the medication as prescribed. Therefore, this study likely overestimates treatment adherence.

\section{Conclusions}

Among the Japanese population with ASCVD initiating statins and/or ezetimibe, a considerable proportion discontinued treatment within an average of 100 days. The use of statin doses of higher-than-moderate intensity were rarely observed. Approximately $10 \%$ of patients had possible statin intolerance. Moreover, many patients had recurrent CVEs despite receiving statins and/or ezetimibe. Similar findings were obtained in the primary prevention diabetes cohort; thus, these findings highlight continued unmet medical need among primary and secondary CVE prevention patients in Japan.

\section{Disclosures}

This study was funded by Amgen Inc., Thousand Oaks, CA, USA. S.P.N., J.M. and K.D. are employees of RTI Health Solutions, Research Triangle Park, NC, USA, which has received remuneration from Amgen Inc. P.P.R., Y.Q. and A.B. were employees and stockholders of Amgen Inc. when this study was conducted. H.I. was an employee of Amgen Astellas BioPharma K.K. and stockholder of Amgen Inc. when this study was conducted. K.M.F. is affiliated with Strategic Healthcare solutions LLC and has received remuneration from Amgen Inc. K.K. received research grants from Daiichi-Sankyo, Astellas, Dainippon-Sumitomo, Behlinger-Ingerheim, Bayer, Fuji film, Central Medical, St. Jude Medical and consulting fees (lecture fee, modest) from Astellas-Amgen Inc., Japan.

\section{References}

1. Annual Report on Kokumin-eisei no Douko (Trends for National Hygiene), Japan Health and Welfare Statistics Association 2008; 51 (in Japanese).

2. World Health Organization (WHO). Global health observatory data repository, life expectancy by country http://apps.who.int/ gho/data/view.main.680?lang=en (accessed October 29, 2013).

3. Ueshima H. Explanation for the Japanese paradox: Prevention of increase in coronary heart disease and reduction in stroke. $J$ Atheroscler Thromb 2007; 14: 278-286.

4. Okamura T, Kokubo Y, Watanabe M, Higashiyama A, Ono Y, Miyamoto Y, et al. Triglycerides and non-high-density lipoprotein cholesterol and the incidence of cardiovascular disease in an urban Japanese cohort: The Suita study. Atherosclerosis 2010; 209: $290-294$

5. Teramoto T, Sasaki J, Ishibashi S, Birou S, Daida H, Dohi S, et al. Executive summary of the Japan Atherosclerosis Society (JAS) guidelines for the diagnosis and prevention of atherosclerotic cardiovascular diseases in Japan - 2012 version. J Atheroscler Thromb 2013; 20: 517-523.

6. Katabami T, Murakami M, Kobayashi S, Matsui T, Ujihara M, Takagi S, et al. Efficacy of low-dose rosuvastatin in patients with type 2 diabetes and hypo high-density lipoprotein cholesterolaemia. J Int Med Res 2014; 42: 457-467.

7. Eldor R, Raz IM. American Diabetes Association indications for statins in diabetes. Diabetes Care 2009; 32: 84-91.

8. Morita H, Ishizaka N. In search of an optimal target level of low density lipoprotein cholesterol for secondary prevention among the Japanese population. J Atheroscler Thromb 2016; 23: 397398.

9. Matsuzawa Y, Kita T, Mabuchi H, Matsuzaki M, Nakaya N, Oikawa S, et al. Sustained reduction of serum cholesterol in low-dose 6-year simvastatin treatment with minimum side effects in 51,321 Japanese hypercholesterolemic patients. Circ J 2003; 67: $287-294$.

10. Saito M, Hirata-Koizumi M, Urano T, Miyake S, Hasegawa R. 
A literature search on pharmacokinetic drug interactions of statins and analysis of how such interactions are reflected in package inserts in Japan. J Clin Pharm Ther 2005; 30: 21-37.

11. Arnold FL, Kusama M, Ono S. Exploring differences in drug doses between Japan and Western countries. Clin Pharmacol Ther 2010; 87: 714-720.

12. Wang P. Statin dose in Asians: Is pharmacogenetics relevant? Pharmacogenomics 2011; 12: 1605-1615.

13. Chang CH, Kusama M, Ono S, Sugiyama Y, Orii T, Akazawa M. Assessment of statin-associated muscle toxicity in Japan: A cohort study conducted using claims database and laboratory information. BMJ Open 2013; 3: e002040.

14. Davidson MH. Safety profiles for the HMG-CoA reductase inhibitors: Treatment and trust. Drugs 2001; 61: 197-206.

15. Shepherd J, Vidt DG, Miller E, Harris S, Blasetto J. Safety of rosuvastatin: Update on 16,876 rosuvastatin-treated patients in a multinational clinical trial program. Cardiology 2007; 107: $433-443$.

16. Rowan C, Brinkler AD, Nourjah P, Chang J, Mosholder A, Barrett JS, et al. Rhabdomyolysis reports show interaction between simvastatin and CYP3A4 inhibitors. Pharmacoepidemiol Drug Saf 2009; 18: 301-309.

17. Ahmad Z. Statin intolerance. Am J Cardiol 2014; 113: 1765 1771.

18. Stone NJ, Robinson JG, Lichtenstein AH, Bairey Merz CN, Blum CB, Eckel RH, et al. 2013 ACC/AHA guideline on the treatment of blood cholesterol to reduce atherosclerotic cardiovascular risk in adults: A report of the American College of Cardiology/American Heart Association Task Force on Practice Guidelines. J Am Coll Cardiol 2014; 63: 2889-2934.

19. Bruckert E, Hayem G, Dejager S, Yau C, Begaud B. Mild to moderate muscular symptoms with high-dosage statin therapy in hyperlipidemic patients - the PRIMO study. Cardiovasc Drugs Ther 2005; 19: 403-414.

20. Wei MY, Ito MK, Cohen JD, Brinton EA, Jacobson TA. Predictors of statin adherence, switching, and discontinuation in the USAGE survey: Understanding the use of statins in America and gaps in patient education. J Clin Lipidol 2013; 7: 472-483.

21. Miyauchi K, Ray K. Review of statin use in patients with acute coronary syndrome in Western and Japanese populations. $J$ Int Med Res 2013; 41: 523-536.
22. Kishimoto I, Makino H, Ohata Y, Tamanaha T, Tochiya M, Anzai T, et al. Intensity of statin therapy and new hospitalizations for heart failure in patients with type 2 diabetes. BMJ Open Diabetes Res Care 2015; 3: e000137.

23. Quek RGW, Fox KM, Wang L, Li L, Gandra SR, Wong N. Lipid lowering treatment trends among diabetes patients with very high cardiovascular disease risk: A real-world study. Poster presented at the 2015 meeting of the American Association of Clinical Endocrinologists; May 13-17, 2015. Nashville, TN, USA.

24. Kim HS, Wu Y, Lin SJ, Deerochanawong C, Zambahari R, Zhao L, et al. Current status of cholesterol goal attainment after statin therapy among patients with hypercholesterolemia in Asian countries and region: The Return on Expenditure Achieved for Lipid Therapy in Asia (REALITY-Asia) study. Curr Med Res Opin 2008; 24: 1951-1963.

25. Teramoto T, Kashiwagi A, Mabuchi H; J-Lap Investigators. Status of lipid-lowering therapy prescribed based on recommendations in the 2002 report of the Japan Atherosclerosis Society Guideline for Diagnosis and Treatment of Hyperlipidemia in Japanese Adults: A study of the Japan Lipid Assessment Program (J-LAP). Curr Ther Res Clin Exp 2005; 66: 80-95.

26. Hata Y, Mabuchi H, Saito Y, Itakura H, Equsa G, Ito H, et al; Working Committee on JAS Guideline for Diagnosis and Treatment of Hyperlipidemia. Report of the Japan Atherosclerosis Society (JAS) guideline for diagnosis and treatment of hyperlipidemia in Japanese adults. J Atheroscler Thromb 2002; 9: $1-27$.

27. Davis KL, Meyers J, Zhao Z, McCollam PL, Murakami M. Dose titration, persistence, and adherence to statin therapy among patients with high-risk vascular disease in Japan. Value Health 2014; 17: A763.

28. Bhatt DL, Eagle KA, Ohman EM, Hirsch AT, Goto S, Mahoney EM, et al. Comparative determinants of 4-year cardiovascular event rates in stable outpatients at risk of or with atherothrombosis. JAMA 2010; 304: 1350-1357.

29. Daida H, Miyauchi K, Ogawa H, Yokoi H, Matsumoto M, Kitakaze M, et al. Management and two-year long-term clinical outcome of acute coronary syndrome in Japan. Circ J 2013; 77: 934-943. 